\title{
Demonstration via Simulation of Stable Confinement of Sheet Electron Beams Using Periodic Magnetic Focusing
}

\author{
John H. Booske, Senior Member, IEEE, and Mark A. Basten
}

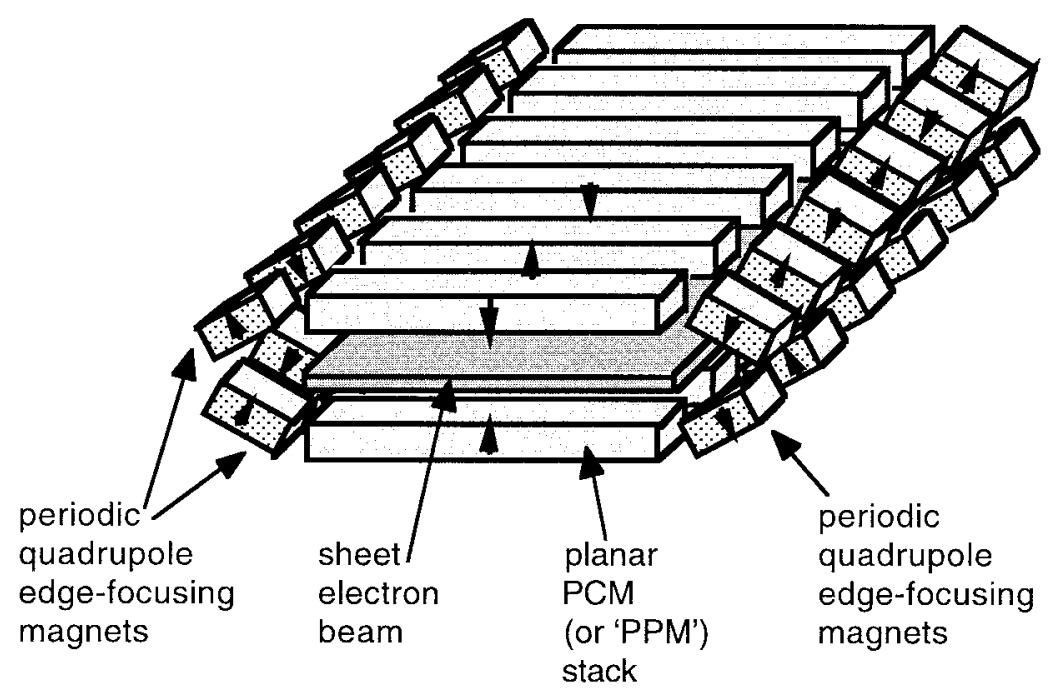

Fig. 1. PCM-PQM magnetic array hybrid for two-plane focusing of sheet electron beams. Arrows show the alternating cusped magnetic polarity of the central PCM section (equivalent to a planar PPM structure) and the alternating quadrupolar polarities of the edge magnets.

\begin{abstract}
Using a two and one-half dimensional (twodimensional for fields, three-dimensional for particle velocities) particle-in-cell (PIC) code we simulate the dynamics of a highly-elliptic sheet electron beam focused by a periodicallycusped-magnetic (PCM) field array. For edge-focusing, a periodic-quadrupole-magnetic (PQM) array is placed along the sides. Very high-space-charge, low-voltage beams may be focused in this way, without disruptive diocotron instability. The PCM-PQM hybrid array appears convenient for twoplane matching of the beam to minimize beam ripple in both transverse dimensions.
\end{abstract}

Index Terms-High power microwave devices, PCM focusing, periodic magnetic focusing, PIC code simulation, PPM focusing, sheet electron beams.

$\mathbf{O}$ $\mathrm{NE}$ of the problems in the scaling of high-power vacuum microwave sources to higher frequencies is the need to transport beams with high space-charge density, since the RF circuit transverse dimensions tend to decrease with wavelength. The use of sheet electron beams can alleviate this difficulty since large amounts of current can be transported, at

Manuscript received May 1, 1998; revised August 24, 1998. This work was supported by the Office of Naval Research (administered by the Vacuum Electronics Branch, Naval Research Laboratory), the Tri-Services Vacuum Electronics Initiative (administered by AFOSR), and by a National Science Foundation Presidential Young Investigator Award.

J. H. Booske is with the Electrical and Computer Engineering Department, University of Wisconsin, Madison, WI 53706 USA (e-mail: booske@engr.wisc.edu).

M. A. Basten is with Northrop Grumman Corporation, Rolling Meadows, IL 60008 USA.

Publisher Item Identifier S 0093-3813(99)02421-2. reduced space-charge density, by increasing the width of the beam. Thus, focusing problems and space-charge debunching effects are avoided and, in addition, the RF power density is reduced due to the extended nature of a rectangular or planar RF interaction circuit. Historically, the main disadvantage of using sheet electron beams is the susceptibility to beam distortion and break up due to the diocotron instability, at least in solenoidal focusing schemes.

Previously, stable magnetic confinement of sheet electron beams without disruptive diocotron instability has been illustrated by numerical simulation for semi-infinite sheet beams in periodically-cusped magnetic (PCM) focusing fields [1], [2]. Theoretical analyses [3] have also suggested that confinement of finite-width (i.e., including edge effects), high-space-charge sheet electron beams might be possible using an "offsetpole" PCM configuration. This offset-pole PCM array is analogous to an offset-pole planar "wiggler" system that was experimentally demonstrated to be effective at two-plane confinement of very low density (negligible space charge) high-voltage sheet electron beams [4].

Here we provide evidence obtained from numerical simulations of an alternative periodic magnetic configuration that stably confines high-space-charge, low-voltage, finite-width sheet electron beams and is considered superior to the offsetpole PCM. The particular magnetic configuration consists of a hybrid combination of a PCM array in the center and a canted, periodic quadrupole magnetic (PQM) array for the sides for edge focusing of the sheet beam, illustrated in Fig. 1. This configuration has several superior features for sheet 

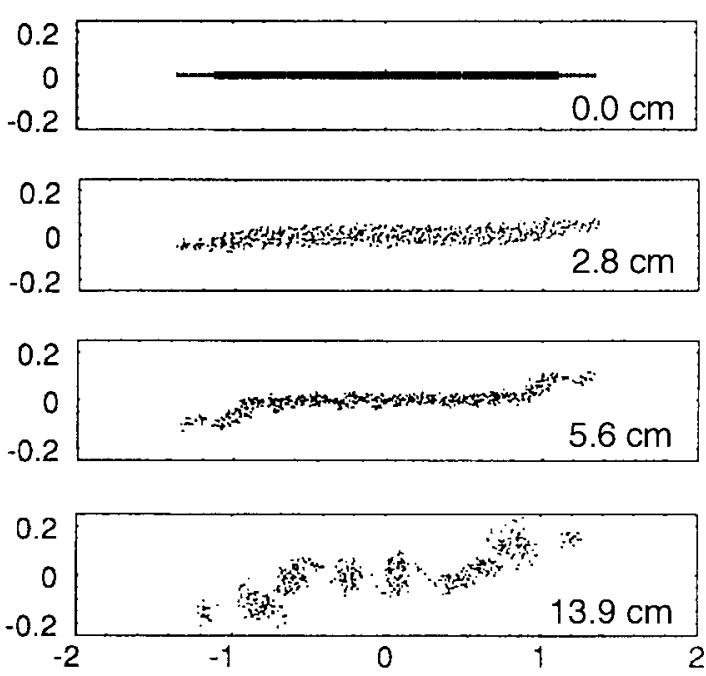

(a)
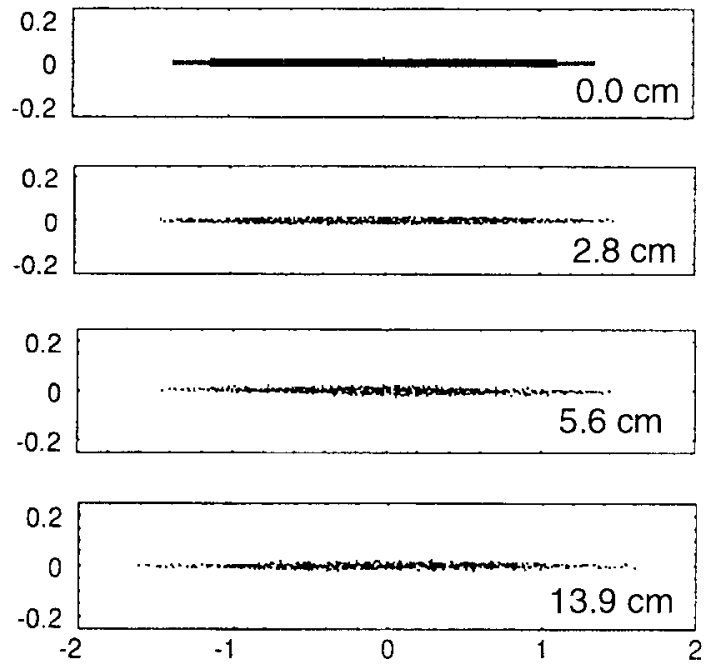

(b)

Fig. 2. Cross-sectional ( $x-y$ plane) images illustrating confinement of sheet electron beams by (a) a contant axial magnetic field and (b) a PCM-PQM periodic magnetic field. The inset numbers indicate distances along the axial or " $z$ " dimension. The numbers along horizontal and vertical axes represent " $x$ " and " $y$ " dimensions, respectively, in centimeters.

beam focusing, including the "strong" focusing characteristic of periodic quadrupole systems [5] as well as flexibility to separately "tune" the edge-focusing fields by varying the side magnet cant angle or spacing independent of the central PCM array.

To investigate the dynamics of sheet beams focused by a PCM-PQM hybrid magnetic field, we used the two and onehalf dimensional particle-in-cell (PIC) code MAGIC [6]. The lack of a third dimension was finessed by using a paraxial assumption and transforming the beam axis or " $z$ " degree of freedom into the time domain, i.e., $z \rightarrow u_{0} t$, where $u_{0}$ is the (approximately constant) axial speed of the electron beam.

Fig. 2 illustrates results of simulations of a sheet electron beam transport dynamics (specifically, the beam has a highlyelliptic cross section) with major and minor radii of $x_{b}=1.4$ $\mathrm{cm}$ and $y_{b}=0.025 \mathrm{~cm}$, respectively, with a total beam current $I_{b}=10 \mathrm{~A}$ and a beam energy of $e V_{b}=10 \mathrm{keV}$. The beam was initially loaded with a Gaussian velocity distribution in all three velocity coordinates, with illustrative rms velocity spread ratios of $v_{\mathrm{th}, x} / u_{0}=v_{\mathrm{th}, y} / u_{0}=v_{\mathrm{th}, z} / u_{0}=1 \%$. Conducting wall boundary conditions were invoked at $x= \pm 2 \mathrm{~cm}$ and $y= \pm 0.25 \mathrm{~cm}$.

Fig. 2(b) shows the evolution of the beam cross section subject to periodic magnetic fields characterized by a peakon-axis PCM field amplitude of $B_{0}=1200 \mathrm{G}$ and a magnet period of $\lambda_{m}=3 \mathrm{~mm}$. No diocotron instability is observed. The independent tunability of the PQM side fields enables matching of the beam in the wide transverse dimension, minimizing overall beam ripple [7]. For comparison, Fig. 2(a) shows the same beam in a static, uniform solenoidal magnetic field $(1200 \mathrm{G})$ oriented along the $z$ axis (pointing out of the page). It is evident that the beam begins to shear and tilt early in the simulation and eventually filaments due to diocotron instability in a fairly faithful reproduction of the same phenomenon observed experimentally in [8].

To emphasize the enhanced capability for high-power, highspace-charge beam transport to be realized with PCM-focused sheet beams, we note that the total perveance of this beam is a considerable $I_{b} / V_{b}^{3 / 2}=10 \mu$ pervs! The current, space charge, and particle densities of the beam correspond to 90.9 $\mathrm{A} / \mathrm{cm}^{2}, 15.5 \mathrm{mC} / \mathrm{m}^{3}$, and $9.7 \times 10^{16} \mathrm{~m}^{-3}$, respectively.

\section{REFERENCES}

[1] J. H. Booske, A. H. Kumbasar, and M. A. Basten, "Periodic focusing and ponderomotive stabilization of sheet electron beams," Phys. Rev. Lett., vol. 71, no. 24, pp. 3979-3982, 1993.

[2] J. H. Booske, M. A. Basten, A. H. Kumbasar, T. M. Antonsen Jr., S. W. Bidwell, Y. Carmel, W. W. Destler, V. L. Granatstein, and D. J. Radack, "Periodic magnetic focusing of sheet electron beams," Phys. Plasmas, vol. 1, no. 5, pp. 1714-1720, 1994.

[3] J. H. Booske, B. D. McVey, and T. M. Antonsen Jr., "Stability and confinement of nonrelativistic sheet electron beams with periodic cusped magnetic focusing," J. Appl. Phys., vol. 73, no. 9, pp. 4140-4155, 1993.

[4] Z. X. Zhang, V. L. Granatstein, W. W. Destler, S. W. Bidwell, J. Rodgers, S. Cheng, T. M. Antonsen Jr., B. Levush, and D. J. Radack, "Experimental and numerical studies of sheet electron beam propagation through a planar wiggler magnet," IEEE Trans. Plasma Sci., vol. 21, pp. 760-767, Dec. 1993.

[5] A. M. Clogston and H. Heffner, "Focusing of an electron beam by periodic fields," J. Appl. Phys., vol. 25, no. 4, pp. 436-447, 1954.

[6] B. Goplen, L. Ludeking, D. SMithe, and G. Warren, "User-configurable MAGIC for electromagnetic PIC calculations," Comput/ Phys. Commun., vol. 87, pp. 54-86, 1995.

[7] M. A. Basten, "Formation and transport of high-perveance electron beams for high-power, high-frequency microwave devices," Ph.D. dissertation, University of Wisconsin-Madison, Madison, WI, 1996.

[8] C. C. Cutler, "Instability in hollow and strip electron beams," J. Appl. Phys., vol. 27, no. 9, pp. 1028-1029, 1956. 